\title{
Reply to: comment on "Is there a relationship between environmental factors and pityriasis rosea?
}

\author{
${\text { Mehak Singh }{ }^{1} \text {, Manoj Pawar }}^{凶}$, Antonio Chuh³ ${ }^{3}$ Vijay Zawar
}

Received: 24 July 2019 | Accepted: 26 September 2019

\section{To the Editor:}

We read with appreciation the comments and speculations presented by Drago et al. (1). We would like to draw your attention to the fact that this study was carried out in the humid subtropical climate of Guwahati (Köppen climate classification Cwa), which has broad temperature, precipitation, and humidity variations coupled with a kaleidoscope of genetic and ethnic diversity.

In a study by Chuh (2), a large number of autoantibodies were identified in the sera of patients with pityriasis rosea (PR) in contrast to the sera of controls, which forms the basis of the explanation that follows.

Our study highlights the Trojan horse phenomenon (3), or antibody-dependent enhancement seen in the development of a rash that results in a secondary autoimmune response in the form of PR rash, which differs significantly from that of either the acute exanthem in dengue fever (Herman's rash) or the secondary maculopapular rash in terms of the time frame as well as clinical presentation. According to this, the virus persists sub-clinically and festers, leading to formation of autoantibodies to the virus. We postulate that these autoantibodies may be responsible for the epidermotropic attack on the skin. The development of such autoantibodies is not subject to a specific timeline. However, we do agree with Drago et al. that the histopathological and immunopathological evidence is amiss. Our first instinct was also to attribute this phenomenon to dengue virus (DENV) immunomodulation permitting the development of such a rash, which again was refuted on the same grounds as pointed out by the author. This hypothesis also underscores the reason why secondary dengue infections hit harder the second time and why dengue hemorrhagic fever has been found to be T-cell mediated as well (4).

Viruses affect antigen presentation and modulate the cytokine milieu, which may in turn play a part in initiation or perpetuation of autoimmunity (5), and such modulation can take place in a timeframe ranging from a few weeks to years.

In 1997, Drago et al. (6) first suggested the role of human herpesvirus 7 ( $\mathrm{HHV} 7$ ) in the etiology of PR by detecting HHV 7 in peripheral blood mononuclear cells and plasma of patients with $\mathrm{PR}$ and not in controls, thus suggesting a probable causal relationship. However, subsequent studies could not find any association between HHV 7 and PR. These correlations may therefore have an underlying ecological fallacy or a genetic predisposition in a particular geographical area. A reactivation of HHV 6 by HHV 7 has been postulated to be the culprit in the development of PR rash (7), and this may also be the case here, whereby DENV might reactivate the same.

In response to the question regarding T-cell mediation of PR etiopathogenesis, we would like to cite the example of Theiler's murine encephalomyelitis virus (TMEV)-induced encephalomyelitis (8). According to this model, lymphocyte subtypes play a paradoxical role in the TMEV disease process; that is, they participate in virus clearance during the acute phase of the disease and aggravate the demyelinating process in the chronic phase of the disease (8). In view of these findings, it might be hypothesized that an extensive peripheral priming of polyfunctional cross-reactive T cells during symptomatic primary DENV infection due to high viral loads and continuous restimulation could potentially establish and maintain a distinct repertoire of virus-specific $\mathrm{T}$ cells, which might lead to epitope spreading and could create a predisposition to PR development (9). Genetic factors, such as the major histocompatibility complex haplotype, are not only directly responsible for disease susceptibility, but will also profoundly influence the antiviral immune response (9).

In a study by Han et al. (10), a time lag of 4 to 6 months was observed between $\mathrm{H} 1 \mathrm{~N} 1$ viral infection and onset of narcolepsy. This was attributed to minimum $80 \%$ cell loss to have a clinical presentation. Although the development of PR rash is not based on this mechanism, such lags are nonetheless not unheard of and should be investigated further for a different underlying mechanism in addition to the previously described mechanisms. As mentioned by the author, this was an incidental finding and we are currently trying to decipher and speculate on the underlying mechanisms of the rash development as well.

Finally, direct proof of causality for viruses to trigger autoimmunity is a challenge due to a variety of reasons; for example, temporal association. That is, the time since the onset of the clinical disease might be so remote from the trigger factor that it becomes too difficult to establish causality. Furthermore, at the time of diagnosis, easily detectable traces of a causative pathogen might no longer exist in the host's system (11).

We would like to challenge the statement that the timeline does not coincide with the development of PR rash; instead, it highlights and supports the Trojan horse phenomenon of autoimmunity.

\section{References}

1. Drago F, Ciccarese G, Parodi A. Is there a relationship between environmental factors and pityriasis rosea? Acta Dermatovenerol Alp Pannonica Adriat. 2019;4: 187.

2. Chuh AA. A prospective case control study of autoimmune markers in patients with pityriasis rosea. Clin Exp Dermatol. 2003;28:449-50. 
3. Huang HW, Tseng HC, Lee CH, Chuang HY, Lin SH. Clinical significance of skin rash in dengue fever: a focus on discomfort, complications, and disease outcome. Asian Pac J Trop Med. 2016;9:713-8.

4. Mongkolsapaya J, Dejnirattisai W, Xu XN, Vasanawathana S, Tangthawornchaikul N, Chairunsri A, et al. Original antigenic sin and apoptosis in the pathogenesis of dengue hemorrhagic fever. Nat Med. 2003;9:921-7.

5. Perl A. Mechanisms of viral pathogenesis in rheumatic disease. Ann Rheum Dis. 1999;58:454-61.

6. Drago F, Ranieri E, Malaguti F, Battifoglio ML, Losi E, Rebora A. Human herpes virus 7 in patients with pityriasis rosea. Electron microscopy investigations and polymerase chain reaction in mononuclear cells, plasma and skin. Dermatology. 1997;195:374-8.
7. Watanabe T, Kawamura T, Jacob SE, Aquilino EA, Orenstein JM, Black JB, et al. Pityriasis rosea is associated with systemic active infection with both human herpesvirus-7 and human herpesvirus-6. J Invest Dermatol. 2002;119:793-7.

8. Grigoriadis N, Hadjigeorgiou GM. Virus-mediated autoimmunity in multiple sclerosis. J Autoimmune Dis. 2006;3:1.

9. Getts DR, Chastain EM, Terry RL, Miller SD. Virus infection, antiviral immunity, and autoimmunity. Immunol Rev. 2013;255:197-209.

10. Han F, Lin L, Li J, Dong SX, An P, Zhao L, et al. HLA-DQ association and allele competition in Chinese narcolepsy. Tissue Antigens. 2012;80:328-35.

11. Christen U, von Herrath MG. Infections and autoimmunity-good or bad? J Immunol. 2005;174:7481-6. 